\title{
On the Rigidity of the $a$-Helix as Measured by Dielectric Studies
}

\author{
Akiyoshi Wada and Hiroshi KrHara \\ Department of Physics, Faculty of Science, The University \\ of Tokyo, Hongo, Tokyo, Japan.
}

(Received October 17, 1971)

\begin{abstract}
The persistence length and flexural rigidity of the $\alpha$-helix of poly $(\gamma-$ benzyl-L-glutamate) have been found to be $1000 \pm 100 \AA$ and $4 \pm 0.4 \times 10^{-19} \mathrm{dyn} / \mathrm{cm}^{2}$, respectively, from a dielectric study in $m$-cresol. Since the electric dipole moment of the $\alpha$-helix is expected to be proportional to the end-to-end distance, flexural rigidity can be estimated in the theoretical framework of the stiff-chain model. The effect of side-chain moment is also discussed.
\end{abstract}

KEY WORDS Polypeptide / $\alpha$-Helix / Dielectric Dispersion / Flexural Rigidity / Dipole Moment /

Several experimental observations of the solution properties of the $\alpha$-helix of polypeptides have led to the belief that the structure is fairly rigid in some solvents, such as ethylene dichloride (EDC), chloroform, dimethylformamide or $m$-cresol. In these solvents, chain extensions measured by light scattering ${ }^{1,2}$ for the radius of gyration, by hydrodynamic properties, ${ }^{1,2}$ such as intrinsic viscosity and sedimentation velocity, and by dielectric properties ${ }^{3-5}$ all have shown a linear dependence on the degree of polymerization of the chain. A rigid-rod model, therefore, seemed to be suitable in these cases.

The idea of flexible nature of the $\alpha$-helix had already been involved in the early work by Doty, et al., ${ }^{1}$ who pointed out a downward deviation from a linear relation between logarithms of intrinsic viscosity and logarithms of molecular weight at the highest extreme of the molecular weight $(M W=800000)$.

Moha, et al., ${ }^{6}$ have shown that the worm-like chain described by Kratky and Porod ${ }^{7}$ is a better model for a high molecular weight $\alpha$-helix. Moha, et al., then deduced a persistence length of $875 \pm 100 \AA$ from light scattering experiments. Recently, Fujita, et al., ${ }^{2}$ have performed a careful and detailed study on this subject using $\operatorname{poly}(\gamma$-benzyl-L-glutamate) (PBLG) in dimethylformamide, using light scattering, sedimentation and viscosity measurements. They concluded that the model used by Moha, et al., may not be unique when the polydispersity in the molecular weight is taken into account. By assuming the $\alpha$-helical conformation found to be correct by Parry and Elliot ${ }^{8}$ from analysis of small-angle X-ray scattering on PBLG in $m$ cresol, Fujita, et al., proposed a super helical and rigid structure as an alternative. Discussion on the flexibility of the $\alpha$-helix in solution is continuing.

A different approach through dielectric studies is used in our attempt to add quantitative information on the flexural rigidity of the $\alpha$-helix in solution. Since the $\alpha$-helix has a large electric dipole moment and since there is nothing comparable to the contribution of such a large moment to the dielectric character in solution, ${ }^{3}$ the study is expected to disclose a new picture of the rigidity of the $\alpha$-helix.

This report is also written to give a reference for the study of the polypeptides which undergoes a conformation transition. Especially, $\operatorname{poly}(\beta-$ benzyl-L-aspertate), which has a transition point to random coil at about $56^{\circ} \mathrm{C}$, has been found to show a drastic change in the dielectric dispersion curve with temperature. ${ }^{9}$

\section{FLEXIBILITY AND DIPOLE MOMENT OF A STIFF CHAIN}

Dielectric studies of PBLG in solution have shown that the orientation of dipole moments 
located in peptide groups are nearly parallel to the helical axis. ${ }^{3}$ Therefore, the total dipole moment, $\boldsymbol{M}$, of the polymer is the vectorial sum of the peptide units, that is,

$$
\boldsymbol{M}=\sum_{i=1}^{n} \boldsymbol{\mu}_{i}=\mu_{0} \sum_{i=1}^{n} \boldsymbol{e}_{i}
$$

where $\mu_{i}$ is the electric dipole vector of the $i$-th unit, $\mu_{0}$, the magnitude of the dipole moment, $\mu_{0}=\left|\boldsymbol{\mu}_{i}\right|$, is 3.4 Debye, $^{3}$ and $\boldsymbol{e}_{i}$ is a unit vector along the axis of the $\alpha$-helix at $i$-th unit. In analogy with the derivation of end-to-end distance, ${ }^{10} \boldsymbol{R}=l_{0} \sum \boldsymbol{e}_{i}\left(l_{0}\right.$, bond length of the unit), the mean square electric dipole moment of a stiff chain can be written as

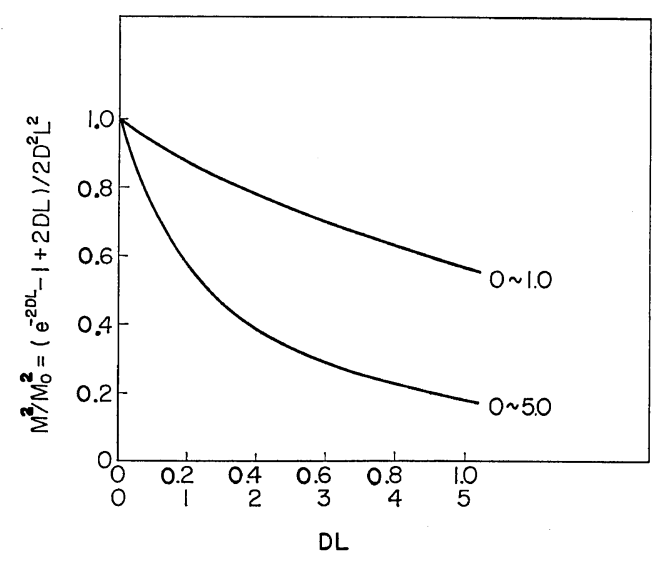

Figure 1. Decrease in total electric dipole moment of a stiff chain plotted against reduced variable $D L . \quad D^{-1}$ represents Kuhn's statistical length, and $L$ represents contour length.

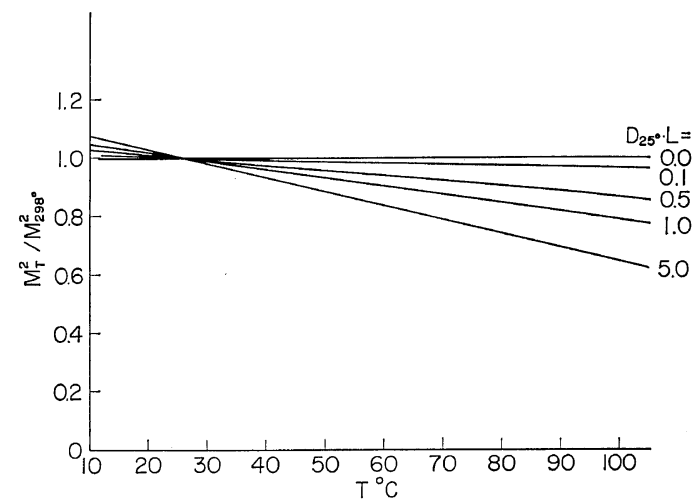

Figure 2. Change of total electric dipole moment of a stiff chain with temperature. $298^{\circ} \mathrm{K}$ is taken as a reference temperature.

$$
\left\langle\boldsymbol{M}^{2}\right\rangle=n^{2} \mu_{0}^{2}\left[\left(\mathrm{e}^{-2 D L}-1+2 D L\right) / 2 D^{2} L^{2}\right]
$$

where $n$ is the degree of polymerization, $D^{-1}$ is the Kuhn statistical length $(=2$ times persistence length): $D=k T / 2 \varepsilon$, with $\varepsilon$ being the flexural rigidity, and $L$ is the contour length of the chain. In terms of a reduced variable, $D L$, and of a dipole moment of a rigid extreme of the chain, $M_{0}=n \mu_{0}$, decrease in the mean square dipole moment $M^{2}$ relative to $M_{0}^{2}$ is shown in Figure 1. The same kind of plot against temperature is shown, also with $D L$ as a parameter, in Figure 2. In the latter figure, $298^{\circ} \mathrm{K}$ has been chosen as a reference temperature.

\section{EXPERIMENTAL}

\section{Material}

PBLG used in the present experiment was prepared by polymerizing $\gamma$-benzyl- $N$-carboxy-Lglutamate anhydride in dioxane. Sodium methoxide was used as an initiator at $A / I=200$. The molecular weight of the sample was estimated from intrinsic viscosity in dichloroacetic acid, with the intrinsic viscosity-molecular weight relationship of Doty-Bradbury-Holtzer. ${ }^{1}$ The degree of polymerization thus obtained was 350 .

$m$-Cresol was dried over $\mathrm{P}_{2} \mathrm{O}_{5}$ and vacuum distilled. Other solvents were dried over $\mathrm{CaCl}_{2}$ and fractionally distilled.

\section{Dielectric Bridge}

The dielectric constant of solvents and solutions were measured by a transformer bridge (Ando Denki Co., Tokyo, Japan) in the frequency range from 300 to $3 \mathrm{MHz}$. A platinum concentric cylinder with the air capacitance of $3.97 \mathrm{pF}$ was used as a liquid cell and calibrated with ethylene dichloride. The liquid cell is kept in a Dewar vessel containing a transformer oil, the temperature of which was maintained to within about $\pm 0.01^{\circ} \mathrm{C}$ over an extended period of time. Measurements of capacitance were carried out by the substitution method. ${ }^{11}$

\section{RESULTS AND DISCUSSIONS}

The data obtained were treated by the method described elsewhere. ${ }^{3,11}$ With dielectric constants of solvent and solutions of known concentrations, specific polarizations of PBLG in $m$-cresol at several temperatures were calculated and were 


\section{A. WAdA and H. KiharA}

plotted against frequency $\nu$ in Figure 3. The reasons for selecting $m$-cresol as a solvent are as follows:

(1) $m$-cresol prevents the intermolecular association which sometimes occurs in helixstabilizing solvents, (2) measurements can be made over a wide temperature range because of its high boiling temperature, and (3) its relatively low conductivity makes precise dielectric measurement possible. In Figure 3, each plot shows a typical dielectric dispersion curve which shifts toward higher frequency as the temperature is raised. To make the results independent of the influence of solvent viscosity, $\eta_{\mathrm{T}}$, the dispersion curves were replotted in Figure 4 in terms of reduced variables $P_{2} \times T / 298$ in ordinate and $\nu \times \eta_{T} \times 298 / T$ in abcsissa, where $\eta_{T}$ is expressed in centipoise. This means that the temperature $\left(298^{\circ} \mathrm{K}\right)$ and viscosity of the solvent ( 1 centipoise) were chosen as a standard state.

All the dispersion data measured at different temperatures come together almost to a single

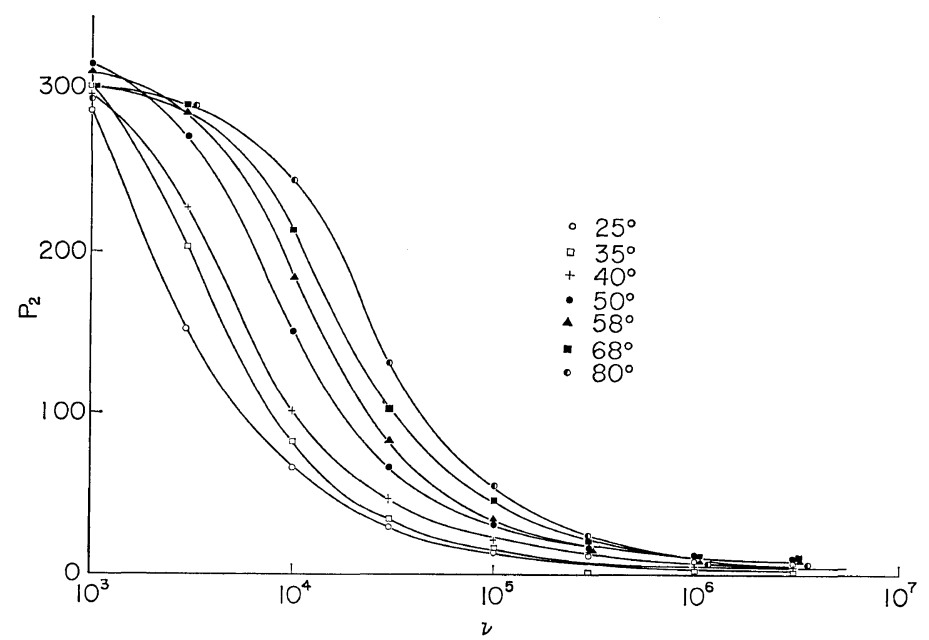

Figure 3. Dielectric dispersion curves of PBLG in $m$-cresol (concn, $50.0 \mathrm{mg} / 10 \mathrm{~m} l$ ).

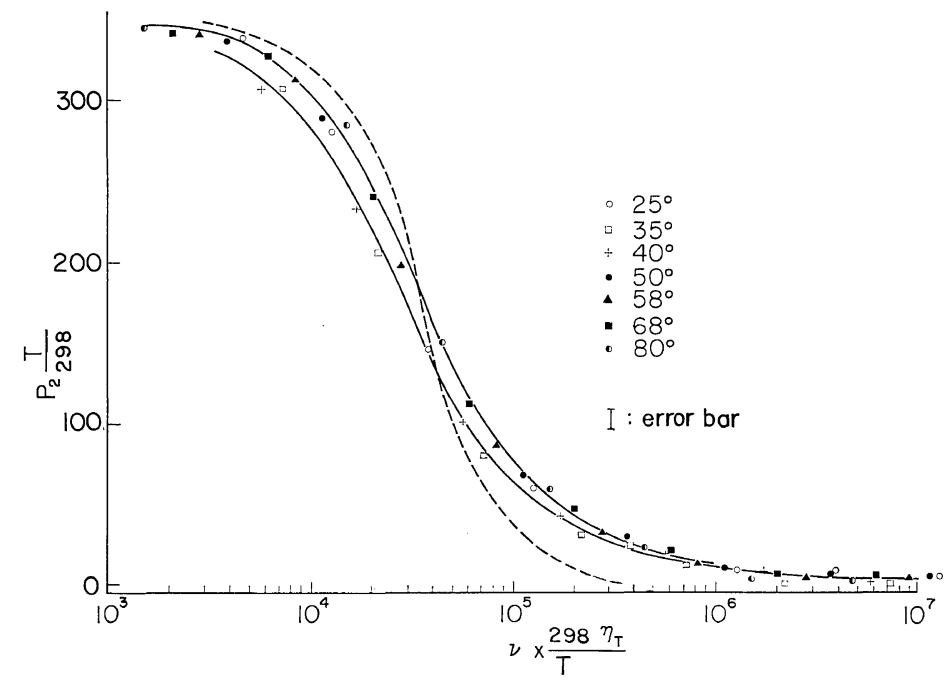

Figure 4. Reduced plots of dispersion curves shown in Figure 3. Two solid lines indicate upper and lower limit of the plot. Broken line indicates Debye dispersion curve of single relaxation time. 
dispersion curve after this reduction process, as shown in Figure 4. This means, first, that the PBLG undergoes no conformational transition in $m$-cresol in the temperature range used. This is in contrast to the case $\operatorname{poly}(\beta$-benzyl-Laspertate) in $m$-cresol, which showed a drastic change of dielectric behavior when the temperature was changed from 40 to $60^{\circ} \mathrm{C}^{9,12}$ If the dipole moment in the backbone chain is the source of the large $P_{2}$, static polarization $P_{2}{ }^{0}$ is found to be almost constant in the range of temperature examined, which implies a rigid helix. Since $P_{2}{ }^{0}$ is proportional to $\boldsymbol{M}^{2}$, the value $D_{298} L$ in eq 2 appears to be smaller than 0.1 when compared with the plot shown in Figure 2. The length $L$ of the present polymer is $525 \AA$, and the persistence length should be longer than $2600 \AA$. This gives $\varepsilon=10.4 \times 10^{-19} \mathrm{dyn} / \mathrm{cm}^{2}$ as a lower limit of the flexural rigidity. There is, however, one possible source that might compensate for the possible decrease of the moment of backbone chain with temperature. It is the contribution of the side chains.

The dipole moments located on the side chains of the $\alpha$-helix of PBLG have been known to contribute negatively to those of the backbone chain. $^{3}$ When the temperature is increased, the movement of side chains should be enhanced, and their negative contribution to the dipole moment decreases, thus resulting in a larger moment. Although the lack of pertinent knowledge about the internal energy of the side chain in solution makes it difficult to go into detail, even a simple model gives some idea of this effect, as can be seen in the following discussion.

Let us consider a side chain whose dipole moment $\mu_{\mathrm{s}}^{0}$ can rotate around the axis perpendicular to the axis of the $\alpha$-helix, as shown in Figure 5. If a single minimum function

$$
E=E_{0} \cos \theta,
$$

is assumed for the potential energy $E$, one can show that the average moment component parallel to the helix axis is given by

$$
\begin{aligned}
\mu_{\mathrm{s}} & =\mu_{\mathrm{s}}^{0} \int_{0}^{\pi} \cos \theta \mathrm{e}^{\beta E_{0} \cos \theta} \mathrm{d} \theta / \int_{0}^{\pi} \mathrm{e}^{\beta E_{0} \cos \theta} \\
& =\mu_{\mathrm{s}}^{0} I_{1}\left(\beta E_{0}\right) / I_{0}\left(\beta E_{0}\right),
\end{aligned}
$$

where $\beta=-1 / k T, I_{0}(z)$ and $I_{1}(z)$ are modified Bessel functions of 0 -th and first order, respec-

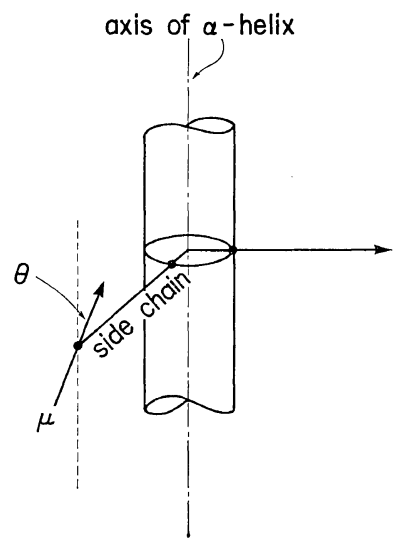

Figure 5. A simplified model and a coordinate system for the rotation of side chain moment, $\mu_{\mathrm{s}}$, indicated by an arrow.

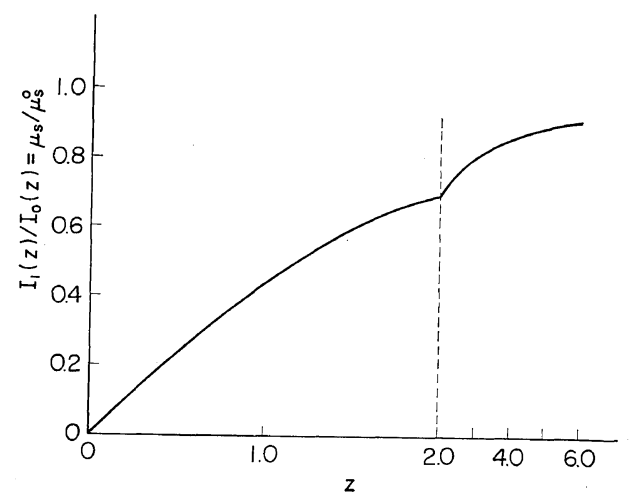

Figure 6. Plot of the function $I_{1}\left(\beta E_{0}\right) / I_{0}\left(\beta E_{0}\right)$ against $\beta E_{0}$.

tively. $\mu_{\mathrm{s}} / \mu_{\mathrm{s}}^{0}$ is plotted against $\beta E_{0}$ in Figure 6. In this figure, the temperature change from 298 to $353^{\circ} \mathrm{K}$ gives rise to a maximum change of $5 \%$ in the dipole contribution of the side chain. As $\mu_{\mathrm{s}}{ }^{0}$ has been estimated as about 2.5 Debye units, ${ }^{3}$ this produces 0.13 Debye increase (from $3.40 \mathrm{D}$ at $298^{\circ} \mathrm{K}$ to $3.53 \mathrm{D}$ at $353^{\circ} \mathrm{K}$ ) in the total moment per residue. Therefore, in Figure 2, the effect of the side chains may lift the value of $M_{\mathrm{T}}^{2} / M_{298}^{2}$ only about $7 \%$, up, or less, at $80^{\circ} \mathrm{C}$. This implies that the acceptable maximum value of $D L$ is 0.25 , which gives $1000 \AA$ as the lowest limit of the persistence length when the compensation effect by the side chain moment is taken into account. Another even larger effect can be considered if the side chains move in a cooperative fashion. Although 
this possibility may not be ruled out completely, it may appear as a drastic change in the specific polarization at the "melting" temperature of side chain order. Thus, this effect must have not been the case in the present experiment. Other effects, such as the reaction field ${ }^{13}$ which reflects the change of the dielectric constant of the environment, does not seem to have produced any major contribution.

Thus, as far as the study on the temperature effect of the dielectric constant is concerened, we can conclude that the persistence length should be longer than $1000 \AA$ A.

At this point, we would like to make comparison with other studies. Marchal and Marchal $^{14}$ have reported a remarkable decrease in dipole moment per residue with degree of polymerization, even at rather low degrees of polymerization, say 100 to 400 , in a chloroformformamide solvent. We have obtained a similar result in this solvent system. The experiments other than dielectric measurement gave results consistent with the rigid model in this solvent system. ${ }^{1}$ Thus, our belief is that the phenomenon is specific to dielectric measurement and does not come from the flexible nature of the $\alpha$ helix. Details about this effect will be reported elswhere. ${ }^{15}$

Tsvetkov, et al. ${ }^{16-18}$ have carried out a series of physical measurements on PBLG in EDC. They obtained persistence lengths of $1100 \pm 100 \AA$ from sedimentation, diffusion and viscosity, $1200 \pm 100 \AA$ from flow birefringence, and $600 \pm$ $100 \AA$ from the Kerr effect. These results, except the one from the Kerr effect, are consistent with the value given above.

The effect of chain flexibility really exists and becomes apparent in high molecular weight samples. Unfortunately, since the dielectric measurement is difficult at frequencies below $100 \mathrm{~Hz}$, it becomes impossible to obtain the specific polarization for high molecular weight samples at the low frequency limit. The Kerr effect is a complementary device suitable for the measurement of slow relaxation of orientation polarization. Ohe, Watanabe, and Yoshioka ${ }^{19}$ have found the effect of chain flexibility both in the dipole moment and the relaxation time of high molecular weight PBLG in $m$-cresol. They kindly allowed us to refer to their results,

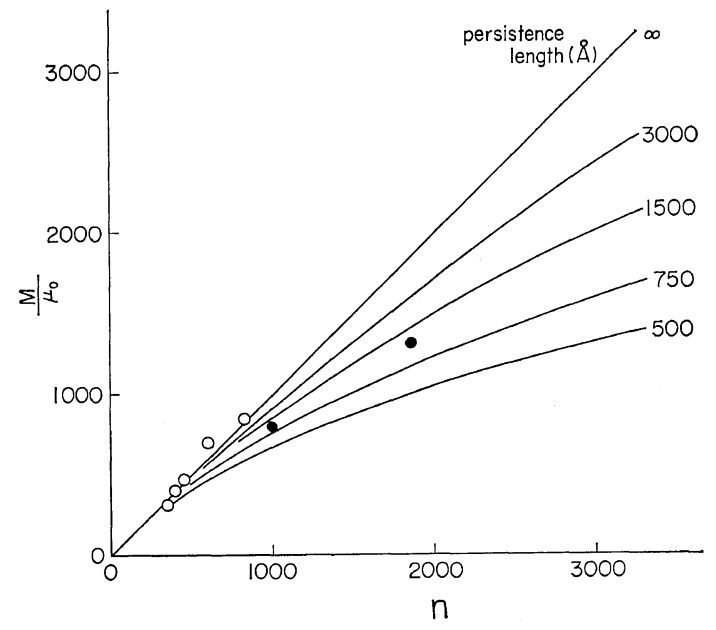

Figure 7. Plots of $M / \mu_{0}$ against degree of polymerization of the $\alpha$-helix. Data obtained by dielectric (O) in EDC and Kerr effect (O) in $m$ cresol measurements are shown.

which are shown in Figure 7 , where $M / \mu_{0}$ are plotted against degree of polymerization. The points obtained from dielectric measurements in $\mathrm{EDC}^{3}$ were also plotted in the same Figure. The results plotted in this Figure also consistent with the conclusion given above.

We should like to mention another approach to the flexibility of the $\alpha$-helix which has been employed in the calculation of internal energy and potential surface of the $\alpha$-helix. Ito and Shimanouchi $^{20}$ have measured the dichroic properties of the far-infrared bands of oriented films of poly(L-alanine) and obtained Young's modulus of $2.31 \times 10^{11} \mathrm{dyn} / \mathrm{cm}^{2}$ by assuming the density of the rod to be $1.24 \mathrm{~g} / \mathrm{cm}^{3}$. Using these values and assuming the $\alpha$-helix to be a hollow cylinder, the radius necessary to produce the flexural rigidity of $4 \times 10^{-19} \mathrm{dyn} / \mathrm{cm}^{2}$ that corresponds to the persistence length of $1000 \AA$ is $4 \AA$. Since the distances from the helix axis to the peptide nitrogen, $\alpha$-carbon, and carbonyl group are $1.57,2.28$, and $1.70 \AA$, respectively, the value obtained above, $4 \AA$, is too large for the cylindrical model equivalent to the $\alpha$-helix. In other words, flexural rigidity estimated from the study of molecular vibration is about five to ten times smaller than the value given above. Direct calculation of the flexural rigidity from bending modes of the $\alpha$-helix of poly(L-alanine) 
has also been made by Ito and Shimanouchi ${ }^{21}$ who obtained the same flexural rigidity and a 140 - $\AA$ persistence length. This value is too small compared to that of PBLG in $m$-cresol. The discrepancy suggests that the side chain of PBLG in $m$-cresol makes PBLG rigid in solution.

The conclusion is that the lower limit of the persistence length and the flexural rigidity of the $\alpha$-helix of PBLG in $m$-cresol is given as $1000 \AA \pm 100 \AA$ and $4 \pm 0.4 \times 10^{-19} \mathrm{dyn} / \mathrm{cm}^{2}$, respectively, from the temperature study of the dielectric constant. On the other hand, the values obtained from other experiments, such as hydrodynamic measurements or the Kerr effect, are found to be around this lower limit. These facts indicate, therefore, that the value given as the lower limit must be the persistence length itself, and that the side chain movement must compensate for a possible decrease in backbonechain moment in raising temperature.

\section{REFERENCES}

1. P. Doty, J. H. Bradbury, and A. M. Holtzer, J. Amer. Chem. Soc., 78, 947 (1956).

2a. H. Fujita, A. Teramoto, K. Okita, T. Yamashita, and S. Ikeda, Biopolymers, 4, 769 (1966).

2b. H. Fujita, A. Teramoto, K. Okita, T. Yamashita, and S. Ikeda, ibid., 4, 781 (1966).

3. A. Wada, "Poly $(\alpha$-amino acid $)$," G. Fasman, Ed., Marcel Dekker, New York, N.Y., 1968, Chapter 9.
4. H. Watanabe, K. Yoshioka, and A. Wada, Biopolymers, 2, 91 (1964).

5. A. Wada, J. Chem. Phys., 30, 328 (1959).

6. P. Moha, G. Weil, and H. Benoit, J. Chim. Phys., 61, 1239 (1964).

7. O. Kratky and G. Porod, Rec. Trav. Chim. Pays-Bas, 68, 1106 (1949).

8. D. A. D. Parry and A. Elliott, Nature, 206, 616 (1965).

9. A. Wada, Chem. Phys. Letters, 8, 211 (1971).

10. N. Saito, K. Takahashi, and Y. Yunoki, $J$. Phys. Soc. Japan 22, 219 (1967).

11. A. Wada, Bull. Chem. Soc. Japan, 33, 822 (1960).

12. A. Wada, T. Tanaka, and H. Kihara, Biopolymers, 11, 587 (1972).

13. A. Wada, J. Chem. Phys., 31, 495 (1959).

14. E. Marchal and J. Marchal, Colloque Ampère, Arch. Sci. Genèver 63, 1961.

15. H. Kihara and A. Wada, to be published.

16. V. N. Tvetkov, Yu. V. Mitin, I. N. Shtennikova, V. R. Glushenkova, G. V. Tarasova, V. S. Skazka, and N. A. Nikitin, Vysokomol. Soedin., 7, 1098 (1965).

17. V. N. Tvetkov, I. N. Shtennikova, E. I. Ryumtsev, and G. I. Okrimenka, ibid., 7, 1104 (1965).

18. V. N. Tsvetkov, I. N. Shtennikova, E. I. Ryumtsev, and V.S. Skazka, ibid., 7, 1111 (1965).

19. H. Ohe, H. Watanabe, and K. Yoshioka, private communication.

20. K. Itoh and T. Shimanouchi, Biopolymers, 9, 383 (1970).

21. K. Itoh and T. Shimanouchi, private communication. 